\title{
Enhancing Precipitation Models by Capturing Multivariate and Multiscale Climate Dynamics
}

\author{
Ruizhou Ding, Dimitrios Stamoulis, Kartikeya Bhardwaj, Diana Marculescu, Radu Marculescu \\ Carnegie Mellon University \\ 5000 Forbes Ave, Pittsburgh, Pennsylvania 15213 \\ \{rding,dstamoul,kbhardwa\}@andrew.cmu.edu,\{dianam,radum\}@cmu.edu
}

\begin{abstract}
To improve precipitation predictions and enable accurate rainfall models for smart water networks, it is imperative to account for multivariate and multiscale precipitation dynamics with long-memory temporal relationships, long-range spatial dependencies, and lowfrequency variability. While prior art has motivated the use of complex networks to capture these trends, existing work is limited to specific climate phenomena (such as El Niño) and regions. In this paper we employ a comprehensive assessment of complex networks with respect to multivariate dynamics across multiple temporal and spatial scales (multiscale). Our work is the first to incorporate both carbon emissions and precipitation anomalies data into a multivariate analysis. By effectively substantiating the ability of complex networks to capture multivariate and multiscale climate dynamics, we postulate their potential as reanalysis and assessment tools to enhance regional water models of arbitrary range and granularity, and to eventually enable reliable smart water systems.
\end{abstract}

\section{CCS CONCEPTS}

•Networks $\rightarrow$ Topology analysis and generation; Network dynamics;

\section{KEYWORDS}

Complex networks, Precipitation, Carbon emissions, Climate anomalies, Complex network properties

\section{ACM Reference format:}

Ruizhou Ding, Dimitrios Stamoulis, Kartikeya Bhardwaj, Diana Marculescu, Radu Marculescu. 2017. Enhancing Precipitation Models by Capturing Multivariate and Multiscale Climate Dynamics. In Proceedings of The 3rd International Workshop on Cyber-Physical Systems for Smart Water Networks, Pittsburgh, PA USA, April 2017 (CySWATER 2017), 4 pages.

DOI: http://dx.doi.org/10.1145/3055366.3055374

\section{INTRODUCTION}

Motivated by the rapidly expanding water crisis [11], several modeling approaches have been fruitfully employed in the context of Smart Water Networks (SWN), aiming to accurately capture water dynamics of water management systems [17] or industrial SWN [18]. These different SWN modeling approaches, wherein a

Permission to make digital or hard copies of all or part of this work for personal or classroom use is granted without fee provided that copies are not made or distributed for profit or commercial advantage and that copies bear this notice and the full citation on the first page. Copyrights for components of this work owned by others than ACM must be honored. Abstracting with credit is permitted. To copy otherwise, or republish, to post on servers or to redistribute to lists, requires prior specific permission and/or a fee. Request permissions from permissions@acm.org.

CySWATER 2017, Pittsburgh, PA USA

(c) 2017 ACM. 978-1-4503-4975-8/17/04 . $\$ 15.00$

DOI: http://dx.doi.org/10.1145/3055366.3055374 key component is the precipitation-related models, naturally yield a trade-off between model complexity and temporal spatial coverage. For instance, precipitation models focusing on specific rivers [2, 4] might be agnostic to the effect of long-term and long-range climate dependencies. This raises an interesting research question for water networks' engineers: given a precipitation anomaly that is recorded in an area adjacent to our model region, to what extent will we need to re-assess the assumptions of our initial model?

Addressing this problem is challenging, since it boils down to capturing the low-frequency, long-memory temporal relationships, and long-range spatial dependencies of global precipitation dynamics $[9,15]$. Recent work has introduced complex networks as means of capturing large-scale meteorological and physical phenomena $[3,12,22,23]$, however their applications to climate and especially in water systems have yet to emerge [20]. Hence, our key goal is to substantiate the usability of complex networks to re-assess the sensitivity of existing small-scale rainfall models to long-term precipitation dynamics, so that the SWN engineers can dynamically decide to re-evaluate any previous model assumptions.

The key contributions in this paper are twofold: (i) to the best of our knowledge, we are the first to capture the correlation between carbon emissions and precipitation anomalies under a complex networks perspective. Our results yield a correlation between air pollution and precipitation anomalies which grows stronger during recent decades. Moreover, (ii) we are the first to employ an extensive analysis that spans multivariate climate dynamics across multiple temporal and spatial scales to demonstrate the ability of complex networks to capture long-memory temporal relationships and long-range spatial dependencies. In conclusion, we postulate the potential of modeling complex precipitation networks as a means to enhance regional, high-granularity SWN models.

\section{RELATED WORK}

Prior art has already motivated the need for multivariable, multiscale methods for capturing temporal relationships and long-term spatial dependencies observed in precipitation dynamics $[9,15]$. Towards this direction, complex networks have emerged as a powerful modeling solution, given their effectiveness in capturing climate trends [19]. Initial attempts have mostly focused on network construction methodologies to identify correlated signals based on statistical significance heuristics and metrics, such as p-value [20], Pearson correlation [12, 23], or correlation strength [10].

By exploiting this analysis foundation [19], state-of-the-art approaches focus on studying climate anomalies using complex networks. Nonetheless, these studies focus on specific physical phenomena, e.g., El Niño [21, 23] and North Atlantic Oscillation [13], 
and limit their multivariate analysis to either ocean- or continentspecific regions $[8,20,21]$. To this end, the key motivation behind our work is to investigate long-term global precipitation dynamics using complex networks. A related work was presented recently [20], however limiting the analysis only to ocean regions, without accounting for the correlation of precipitation dynamics with global air pollution trends. In contrast, we aim to extend our exploration to global, multi-feature climate anomalies by incorporating yearly data for precipitation, temperature, and carbon emissions dating from 1950 to present.

\section{METHODOLOGY}

We base our methodology on climate networks, i.e., complex networks constructed from climate data, wherein the nodes are the grids on the globe, and an edge indicates a significant correlation between a pair of grid points. The weight of an edge indicates the magnitude of the correlation. We use daily precipitable water and temperature data from the NCAR Reanalysis project [16] and monthly carbon emission data from the CDIAC [6]. Since carbon emissions for ocean grid points are unavailable, we focus on land areas with a grid resolution of $2.5^{\circ} \times 2.5^{\circ}$. For each year $y$, the network weights are described by the adjacent matrix $W \in R^{l o n g \times l a t}$, where long $\times$ lat are the grid dimensions for the whole globe. The weights are computed using the Pearson correlation of the yearly time series data at any two grid points. That is, for each year $y$ from 1950 to 1999 , the weight between grid location $i$ and $j$ is:

$$
w_{i, j}^{(y)}=\left|\frac{\left[\boldsymbol{x}_{\boldsymbol{i}}^{(\boldsymbol{y})}-\mu\left(\boldsymbol{x}_{\boldsymbol{i}}^{(\boldsymbol{y})}\right)\right]^{T} \cdot\left[\boldsymbol{x}_{\boldsymbol{j}}^{(\boldsymbol{y})}-\mu\left(\boldsymbol{x}_{j}^{(\boldsymbol{y})}\right)\right]}{\sigma\left(\boldsymbol{x}_{\boldsymbol{i}}^{(\boldsymbol{y})}\right) \cdot \sigma\left(\boldsymbol{x}_{\boldsymbol{j}}^{(\boldsymbol{y})}\right)}\right|
$$

where $\boldsymbol{x}_{\boldsymbol{i}}^{(\boldsymbol{y})}$ is the daily data that span the entire year $y$. The absolute value is taken since both positive and negative correlation coefficients indicate a strong relationship. We convert the time series of precipitable water and temperature to the anomalies data by subtracting the long-term mean, and dividing with the longterm standard deviation computed over all considered years (i.e., the mean of all January 1st days). This normalization is shown to effectively reduce temporal autocorrelation in the time series [20].

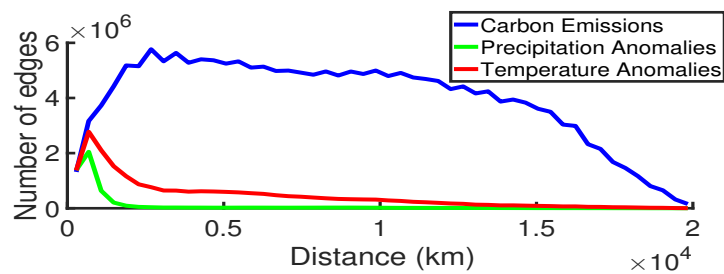

Figure 1: Frequency distribution of edge lengths: our constructed networks correctly capture both proximity-based and long-range correlations. For better visualization of all three features, we connect the centers of the histogram bars.

Finally, we determine the statistically significant correlation values by using the p-value of the correlation result as the significancepruning heuristic. That is, we prune out edges when the $\mathrm{p}$-value of their correlation is more that $10^{-10}$ [20]. To assess the correctness of the generated networks, we plot the frequency distribution of the edge lengths throughout all years in Figure 1. As expected, for all three features we observe the left-most "peak" that corresponds to the close-proximity dependencies and to the right the "tail" of long-distance correlations. Moreover, it is worth noticing the "tail thickness" of carbon emission data that reflects the significantly higher long-range dependence across entire regions of similarly polluted air masses. These results are consistent with prior art [20], and especially the findings related to long-distance "connections", i.e., teleconnections [1].

\section{EXPERIMENTAL RESULTS}

\subsection{Multivariate, Long-memory Temporal Climate Dynamics}

We use the daily data to compute the edges for the global precipitation anomalies, temperature anomalies, and carbon emissions networks. We then compute the average degree for each yearly network for all three features, i.e., the average number of connections between all nodes. Intuitively, a highly-connected network with more correlated nodes capture climate anomalies that affect increasingly wider regions. Figure 2 shows the average degree per network from 1950 to 1999 . For readability purposes, we normalize by the maximum average degree value per curve.

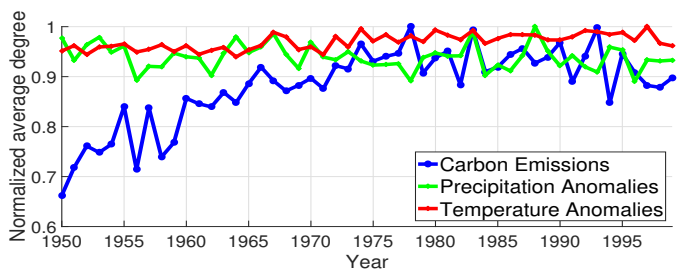

Figure 2: Dynamic evolution of network average degree (1950 - 1999). As expected, precipitation and temperature anomalies exhibit correlation; moreover, our results suggest that the correlation between precipitation anomalies and carbon emissions grows stronger during recent decades.

Based on the dynamic evolution of climate anomalies and air pollution, we have two key observations. First, among the three curves, carbon emissions exhibit stronger increase especially during the last four decades after 1950. In addition, we compute the correlation coefficient between the acquired signals and we find that the correlation between the average degree of carbon emissions and precipitation anomalies grows stronger during more recent decades. That is, while precipitation anomalies and carbon emissions have an overall correlation coefficient of 0.3091 , this value increases to 0.4644 and eventually to 0.6018 the last 30 and 20 years, respectively. As expected, precipitation and temperature patterns have correlated network average degree with an overall coefficient of 0.5368 . This analysis captures the effectiveness of complex networks to highlight long-memory, long-term relationships between climate features.

We further highlight global climate trends by investigating the evolution of network clusters. To this end, we detect communities in all the yearly global networks for the three features, by using the Louvain algorithm [5]. Figure 3 shows the communities detection results for precipitation anomalies (top), carbon emissions (middle), and temperature anomalies (bottom) for the years 1955, 1975, and 


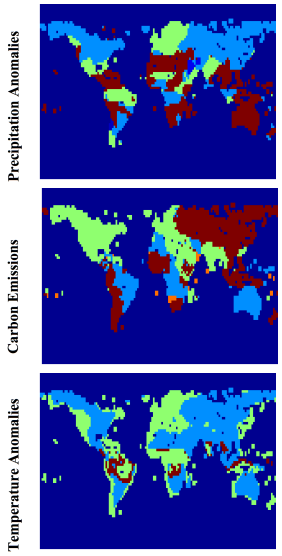

1955
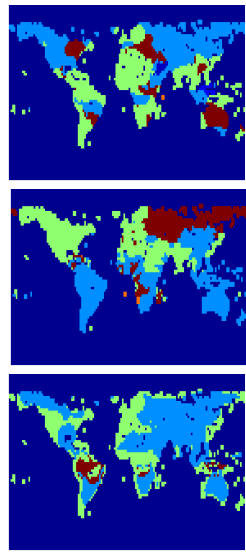

1975
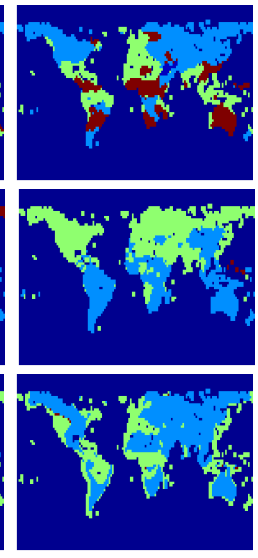

1995
Figure 3: Network clusters (communities) for precipitation anomalies (top), carbon emissions (middle), and temperature anomalies (bottom) for the years 1955, 1975, and 1995. Each color corresponds to one community.

1995. First, similar to prior art studying precipitation and temperature patterns [20], we observe that the clusters illustrate relative stability over time. Nonetheless, we observe that carbon emission clusters increase in size and coverage, suggesting an ever-increasing trend that spans the entire globe.

Unlike prior art on complex climate anomalies networks which do not account for air pollution data, our analysis highlights several interesting observations. First, several regions, such as North America and Europe, belong to the same carbon emission community over time, hence indicating that carbon data have high correlation across entire long-range continental land masses. Moreover, since carbon emission patterns are more likely to be dictated by human activity, the boundaries of carbon communities are closer to the country boundaries, such as United States, India, China, etc., compared to precipitation and temperature anomalies data.

\subsection{Long-range Spatial Precipitation Anomalies}

In the previous Subsection, we exploited the ability of complex networks to capture multivariate dynamics, even under long-term and long-memory dependencies. Next, we investigate the inherent ability of precipitation networks to capture special dependences. We focus on two important properties of complex networks, the degree centrality and the betweenness centrality [7]. The former metric captures the degree of each node, while the latter measures the probability that this node appears in the shortest path between two random nodes in the network. Note that we compute betweenness centrality using unweighted networks. To highlight their evolution, these two metrics for three consecutive years are shown in Figure 4 in log scale. The left and right columns correspond to degree and betweenness centrality, respectively, while red areas have largest centrality values and blue areas lowest ones. The presented years have been arbitrarily selected, since a detailed analysis for node degree throughout all years follows in the next Subsection.

By observing the centers (dark red clusters) of the centrality maps, we make certain key observations. First, the centers in the
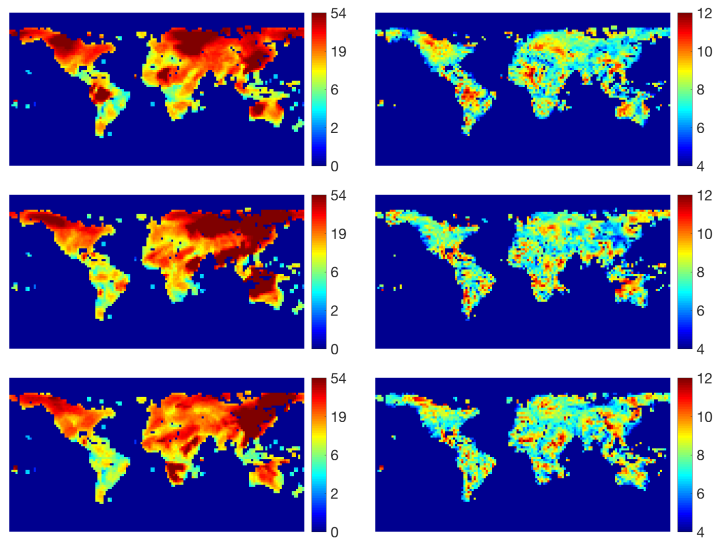

Figure 4: Node degree (left column) and betweenness centrality (right column) of precipitation networks, for the years 1950, 1951, and 1952 (darker colors indicate larger values).

degree centrality map can be interpreted as the most influential nodes [7]. In other words, when they experience a climate anomaly, the nodes connected to them are highly likely to be affected accordingly. Most importantly, it is worth stressing that the centers are aligned in both metrics. This is to be expected, since these centers correspond to locations affected heavily by global jet streams and ocean currents. That is, the highly connected nodes simultaneously exhibit high centrality throughout all the years along the west coast of N. America, of S. America, of N. Europe, of Africa, of Australia, and the east coast of Central America and Asia. From a network perspective, nodes with high betweenness centrality correspond to temporal and spatial shortest paths through which the precipitable water traverses. All these regions are consistent with prior art findings related to polar jet streams [14] and ocean currents [7].

\subsection{Long-memory Multiscale Precipitation Patterns}

In this Subsection, we assess the ability of complex networks to capture inherent multiscale dynamics between precipitation and temperature anomalies. To this end, we employ the analysis for both the entire globe and N. America, and we now focus on quarterly autumn data. That way, we effectively investigate different scales both temporal and spatial. Figure 5 shows the yearly node degree distribution for global precipitation and temperature anomalies networks, as well as the average degree across all the nodes for the entire globe (left) and N. America (right). For each year, the degrees of all the nodes are vectorized as a column in the Figures, and sorted by their values. The color shows the value of node degree (red coloring indicates larger values). Their correlation coefficient for globe and N. America is 0.56 and 0.55 , respectively. This key observation that the average degrees of precipitation and temperature networks are strongly correlated across both scales, demonstrates the multiscale modeling capacities of complex networks. From an applicable point of view, years with more temperature anomalies are also likely to witness precipitation anomalies, and vice versa, a key insight that can be used to tackle sensitivities of regional water models to dynamic climate anomalies. 

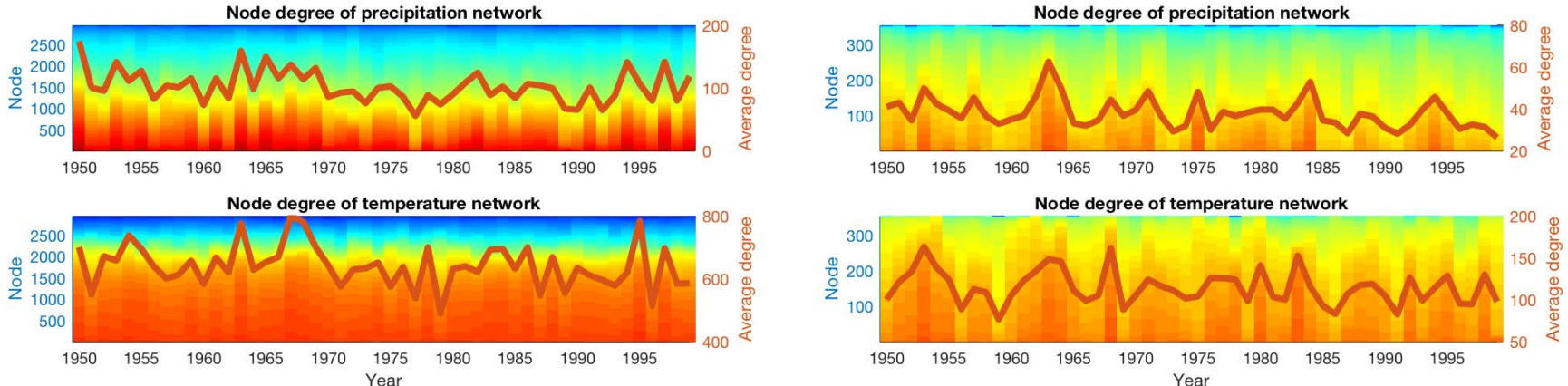

Figure 5: Yearly node degree distribution and the average degree value for precipitation anomalies network and global temperature anomalies network, for the entire globe (left) and N. America (right). For the two cases, the correlation coefficients of the two average degree curves are 0.56 and 0.55 , respectively.

\section{CONCLUSION AND FUTURE WORK}

In this work, we comprehensively evaluate the modeling capacity of complex networks to capture long-memory temporal, large-scale spatial, and multiscale climate dynamics by investigating their properties through the respective case studies. Towards this direction, we are the first to capture the correlation between carbon emissions and precipitation anomalies using complex networks. Moreover, through our key findings, we postulate the potential of complex network approaches as a complementary means to dynamically evaluate the need to re-assess regional water models, and to eventually enhance their accuracy.

In conclusion, our work has motivated that these key results can be flexibly incorporated into fine-grained smart water networks, whose integration we leave for future work. An orthogonal step to our analysis is to train a model on specific water-systems (e.g., a river model $[2,4])$ throughout multiple decades, and to investigate the correlation between the model's coefficients and the characteristics of a complex network spanning a wider region around the point of interest.

\section{ACKNOWLEDGMENTS}

This work was supported in part by the US National Science Foundation (NSF) under CyberSEES Grant CCF-1331804.

\section{REFERENCES}

[1] Michael A Alexander, Ileana Bladé, Matthew Newman, John R Lanzante, NgarCheung Lau, and James D Scott. 2002. The atmospheric bridge: The influence of ENSO teleconnections on air-sea interaction over the global oceans. Fournal of Climate 15, 16 (2002), 2205-2231.

[2] Eram Artinyan, Beatrice Vincendon, Kamelia Kroumova, Nikolai Nedkov, Petko Tsarev, Snezhanka Balabanova, and Georgy Koshinchanov. 2016. Flood forecasting and alert system for Arda River basin. Journal of Hydrology 541 (2016), 457-470.

[3] Kartikeya Bhardwaj and Radu Marculescu. 2015. Network-based modeling and analysis of cloud fraction and precipitation: A case study for the ohio river basin. In Proceedings of the 1st ACM International Workshop on Cyber-Physical Systems for Smart Water Networks. ACM, 12.

[4] Kartikeya Bhardwaj and Radu Marculescu. 2017. K-Hop Learning: A NetworkBased Feature Extraction for Improved River Flow Prediction. In Proceedings of The 3rd International Workshop on Cyber-Physical Systems for Smart Water Networks (CySWATER 2017). 1-4.

[5] Vincent D Blondel, Jean-Loup Guillaume, Renaud Lambiotte, and Etienne Lefebvre. 2008. Fast unfolding of communities in large networks. Fournal of statistical mechanics: theory and experiment 2008, 10 (2008), P10008.
[6] T.A. Boden, G. Marland, and R.J. Andres. 2013. Global, Regional, and National Fossil-Fuel CO2 Emissions. Carbon Dioxide Information Analysis Center, Oak Ridge National Laboratory, U.S. Department of Energy, Oak Ridge, Tenn., U.S.A. (2013).

[7] Jonathan F Donges, Yong Zou, Norbert Marwan, and Jürgen Kurths. 2009. The backbone of the climate network. EPL (Europhysics Letters) 87, 4 (2009), 48007.

[8] Imme Ebert-Uphoff and Yi Deng. 2012. A new type of climate network based on probabilistic graphical models: Results of boreal winter versus summer. Geophysical Research Letters 39, 19 (2012).

[9] Lisa Goddard, Simon J Mason, Stephen E Zebiak, Chester F Ropelewski, Reid Basher, and Mark A Cane. 2001. Current approaches to seasonal to interannual climate predictions. International fournal of Climatology 21, 9 (2001), 1111-1152.

[10] Avi Gozolchiani, Kazuko Yamasaki, O Gazit, and Shlomo Havlin. 2008. Pattern of climate network blinking links follows El Nino events. EPL (Europhysics Letters) 83, 2 (2008), 28005.

[11] Daniel Griffin and Kevin J Anchukaitis. 2014. How unusual is the 2012-2014 California drought? Geophysical Research Letters 41, 24 (2014), 9017-9023.

[12] O. Guez, A. Gozolchiani, Y. Berezin, S. Brenner, and S. Havlin. 2012. Climate network structure evolves with North Atlantic Oscillation phases. EPL (Europhysics Letters) 98, 3 (2012), 38006.

[13] Guez, O., Gozolchiani, A., Berezin, Y., Wang, Y., and Havlin, S. 2013. Global climate network evolves with North Atlantic Oscillation phases: Coupling to Southern Pacific Ocean. EPL 103, 6 (2013), 68006.

[14] Richard Hall, Róbert Erdélyi, Edward Hanna, Julie M Jones, and Adam A Scaife. 2015. Drivers of North Atlantic polar front jet stream variability. International fournal of Climatology 35, 8 (2015), 1697-1720.

[15] Martin P Hoerling, Arun Kumar, and Taiyi Xu. 2001. Robustness of the nonlinear climate response to ENSOfis extreme phases. Fournal of Climate 14, 6 (2001), 1277-1293.

[16] Eugenia Kalnay, Masao Kanamitsu, Robert Kistler, William Collins, Dennis Deaven, Lev Gandin, Mark Iredell, Suranjana Saha, Glenn White, John Woollen, and others. 1996. The NCEP/NCAR 40-year reanalysis project. Bulletin of the American meteorological Society 77, 3 (1996), 437-471.

[17] Luis Montestruque and MD Lemmon. 2015. Globally coordinated distributed storm water management system. In Proceedings of the 1st ACM International Workshop on Cyber-Physical Systems for Smart Water Networks. ACM, 10.

[18] Athanasia Panousopoulou and Panagiotis Tsakalides. 2016. On graph-based feature selection for multi-hop performance characterization in industrial smart water networks. In Cyber-physical Systems for Smart Water Networks (CySWater), 2016 International Workshop on. IEEE, 19-24.

[19] Karsten Steinhaeuser and Nitesh V Chawla. 2010. Identifying and evaluating community structure in complex networks. Pattern Recognition Letters 31, 5 (2010), 413-421.

[20] Karsten Steinhaeuser, Auroop R Ganguly, and Nitesh V Chawla. 2012. Multivariate and multiscale dependence in the global climate system revealed through complex networks. Climate dynamics 39, 3-4 (2012), 889-895.

[21] Anastasios A Tsonis and Kyle L Swanson. 2008. Topology and predictability of El Nino and La Nina networks. Physical Review Letters 100, 22 (2008), 228502.

[22] Anastasios A Tsonis, Kyle L Swanson, and Paul J Roebber. 2006. What do networks have to do with climate? Bulletin of the American Meteorological Society 87, 5 (2006), 585-595.

[23] K. Yamasaki, A. Gozolchiani, and S. Havlin. 2008. Climate Networks around the Globe are Significantly Affected by El Niño. Phys. Rev. Lett. 100 (Jun 2008), 228501. Issue 22 . 